\title{
Activity of the thiopeptide antibiotic nosiheptide against contemporary strains of methicillin-resistant Staphylococcus aureus
}

\author{
Nina M Haste ${ }^{1}$, Wdee Thienphrapa ${ }^{1}$, Dan N $\operatorname{Tran}^{1}$, Sandra Loesgen ${ }^{2,5}$, Peng Sun ${ }^{2}$, Sang-Jip Nam ${ }^{2,4}$, \\ Paul R Jensen ${ }^{2}$, William Fenical ${ }^{2,3}$, George Sakoulas ${ }^{1}$, Victor Nizet ${ }^{1,3}$ and Mary E Hensler ${ }^{1}$
}

The rapid rise in antimicrobial resistance in bacteria has generated an increased demand for the development of novel therapies to treat contemporary infections, especially those caused by methicillin-resistant Staphylococcus aureus (MRSA). However, antimicrobial development has been largely abandoned by the pharmaceutical industry. We recently isolated the previously described thiopeptide antibiotic nosiheptide from a marine actinomycete strain and evaluated its activity against contemporary clinically relevant bacterial pathogens. Nosiheptide exhibited extremely potent activity against all contemporary MRSA strains tested including multiple drug-resistant clinical isolates, with MIC values $\leqslant 0.25 \mathrm{mg} \mathrm{I}^{-1}$. Nosiheptide was also highly active against Enterococcus spp. and the contemporary hypervirulent BI/NAP1/027 strain of Clostridium difficile but was inactive against most Gram-negative strains tested. Time-kill analysis revealed nosiheptide to be rapidly bactericidal against MRSA in a concentration- and time-dependent manner, with a nearly 2-log kill noted at $6 \mathrm{~h}$ at $10 \times$ MIC. Furthermore, nosiheptide was found to be non-cytotoxic against mammalian cells at $>>100 \times$ MIC, and its anti-MRSA activity was not inhibited by $20 \%$ human serum. Notably, nosiheptide exhibited a significantly prolonged post-antibiotic effect against both healthcare- and community-associated MRSA compared with vancomycin. Nosiheptide also demonstrated in vivo activity in a murine model of MRSA infection, and therefore represents a promising antibiotic for the treatment of serious infections caused by contemporary strains of MRSA.

The Journal of Antibiotics (2012) 65, 593-598; doi:10.1038/ja.2012.77; published online 10 October 2012

Keywords: contemporary MRSA; marine actinomycete; nosiheptide; thiopeptide

\section{INTRODUCTION}

Multidrug resistant hospital-associated (HA-) and highly virulent community-associated (CA-) methicillin-resistant Staphylococcus aureus (MRSA) have shown increased tolerance or resistance in recent years to vancomycin, linezolid and daptomycin with accompanying reduction in clinical antimicrobial efficacy. The development of novel agents without cross-resistance to current antimicrobials against MRSA infections is desperately needed. ${ }^{1}$

In our screen of marine-derived actinomycete extract libraries for anti-MRSA activity, we identified a potent fraction derived from strain CNT-373, a Streptomycetes species isolated from a marine sediment collected in Fiji. The 1221.16-mol wt active component was purified and identified by NMR as the thiopeptide antibiotic nosiheptide. Thiopeptide antibiotics encompass a large family of compounds that include thiostrepton and nocathiacin and are comprised of sulfur- and nitrogen-rich heterocycles linked to nonnatural amino acids. ${ }^{2,3}$ Nosiheptide, also referred to historically as multhiomycin, was originally isolated in 1970 and shown to be structurally similar to thiostrepton. ${ }^{4,5}$ Several thiopeptide antibiotics including nosiheptide block protein synthesis by the inhibition of elongation factors Tu and G. ${ }^{6}$ Historically, nosiheptide has been used as a growth-promoting additive in animal feed ${ }^{7}$ but was never developed further as a human therapeutic.

Although the discovery of new chemical scaffolds provides the potential for novel drugs with new mechanisms of action, the re-investigation of previously discovered antibacterial scaffolds also provides a valuable source of compounds with therapeutic potential. Surprisingly, despite the structural identification and description of Gram-positive antibacterial activity, no detailed characterization of nosiheptide activity against contemporary drug-resistant strains such as MRSA has been undertaken. Here, we investigate nosiheptide activity in vitro against a panel of contemporary MRSA and other Gram-positive clinical isolates. MRSA killing kinetics and postantibiotic effects (PAE) are characterized, and nosiheptide in vivo

\footnotetext{
${ }^{1}$ Department of Pediatrics, University of California San Diego, La Jolla, CA, USA; ${ }^{2}$ Center for Marine Biotechnology and Biomedicine, Scripps Institution of Oceanography, University of California San Diego, La Jolla, CA, USA and ${ }^{3}$ Skaggs School of Pharmacy and Pharmaceutical Sciences, University of California San Diego, La Jolla, CA, USA Correspondence: Dr ME Hensler, Department of Pediatrics, University of California San Diego, 9500 Gilman Drive, La Jolla, CA 92093-0687, USA.

E-mail: mhensler@ucsd.edu

${ }^{4}$ Current address: College of Pharmacy, Suncheon National University, Suncheon 540-950, Republic of Korea.

${ }^{5}$ Current address: Laboratory of Bioorganic Chemistry, National Institute of Diabetes and Digestive and Kidney Diseases, National Institutes of Health, Bethesda, MD, USA 20892.

Received 6 June 2012; revised 9 August 2012; accepted 15 August 2012; published online 10 October 2012
} 
activity is demonstrated. Our results indicate that 're-discovered' natural product antibiotics may harbor meaningful therapeutic activity against contemporary multidrug resistant pathogens, and therefore warrant further consideration and preclinical development in hopes of expanding our current limited pharmacological arsenal.

\section{MATERIALS AND METHODS}

Strain isolation and identification

Strain CNT-373 was isolated from a marine sediment sample collected at a depth of $5 \mathrm{~m}$ off Nacula Island, Fiji. The sediment was dried overnight in a laminar flow hood and then stamped on agar plates containing medium Al ( $10 \mathrm{~g}$ starch, $4 \mathrm{~g}$ yeast extract, $2 \mathrm{~g}$ peptone, $16 \mathrm{~g}$ agar and 11 seawater) supplemented with $100 \mathrm{mgl}^{-1}$ cyclohexamide to reduce fungal growth. The strain was identified as a Streptomyces sp. based on 16S rRNA gene sequence analysis (http://eztaxon-e.ezbiocloud.net/ezt_identify) and shares greatest similarity (98.3\%) with the type strain Streptomyces althioticus. This low level of sequence identity suggests it may be a new species. The $16 \mathrm{~S}$ rRNA sequence has been deposited in GenBank under accession number JQ946086.

\section{Fermentation and extraction}

A 2-ml-frozen glycerol stock of strain CNT-373 was used to inoculate $25 \mathrm{ml}$ of liquid $\mathrm{A} 1$ medium and shaken at 230 r.p.m. and $27^{\circ} \mathrm{C}$. After 5 days, the seed culture was used to inoculate a 1-l culture in growth medium Albfe $+\mathrm{C}$ (A1 medium with the addition of $1 \mathrm{~g} \mathrm{CaCO}_{3}, 100 \mathrm{mg} \mathrm{KBr}$ and $40 \mathrm{mg} \mathrm{Fe}_{2}$ (S$\left.\mathrm{O}_{4}\right)_{3} \bullet 4 \mathrm{H}_{2} \mathrm{O}$ ). After 3 days of shaking, 25 -ml aliquots were used to inoculate 18 Fernbach flasks (2.81) each containing 11 medium Albfe + C. After 7 days of cultivation, sterilized XAD-16 resin $\left(20 \mathrm{gl}^{-1}\right)$ was added, shaken for $6 \mathrm{~h}$ and collected by filtration through cheesecloth. The resin was then washed with deionized water and eluted with acetone. The acetone solvent was removed under reduced pressure and the resulting aqueous layer extracted with ethyl acetate $(3 \times 500 \mathrm{ml})$. The combined ethyl acetate extracts were dried over anhydrous sodium sulfate, decanted and concentrated to dryness to yield $2.4 \mathrm{~g}$ of crude material.

\section{Isolation of nosiheptide}

The crude extract was dissolved in methanol and dichloromethane, adsorbed onto silica gel $(2.5 \mathrm{~g})$, and fractionated on a short column of silica gel $(6 \times 2.5 \mathrm{~cm}, h \times w)$ using a $10 \%$ step gradient from $100 \%$ isooctane to $100 \%$ ethyl acetate as eluents. Fractions with antibacterial activity were eluted with 90 and $100 \%$ ethyl acetate. The active fractions were pooled and subjected to a size exclusion column chromatography with Sephadex LH-20 using methanol as eluent. The final purification step was achieved with silicia gel, using ethyl acetate with $10 \%$ dimethylformamide to give nosiheptide ( $210 \mathrm{mg}$ ).

\section{Spectroscopic analysis of nosiheptide}

${ }^{1} \mathrm{H},{ }^{13} \mathrm{C}$ and $2 \mathrm{D}$ NMR spectroscopic data were obtained on a Varian Inova $500-$ $\mathrm{MHz}$ spectrometer in a solvent mixture of methanol- $d_{4}$ and $\mathrm{CDCl}_{3}(3: 1)$ to facilitate solubility. Offline processing was conducted using topspin NMR software by Bruker BioSpin 2011 (iNMR, http://www.inmr.net). The NMR data (Table 1) are in good agreement with previously published NMR data for nosiheptide, ${ }^{8}$ with the resultant nosiheptide structure shown (Figure 1). Highresolution ESI-TOF mass spectra were provided by the MS facility at the Department of Chemistry and Biochemistry at the University of California San Diego, CA: HR-ESI-TOF-MS $[\mathrm{M}+\mathrm{H}]^{+} \mathrm{m} / z 1222.1565$ (calcd for $\mathrm{C}_{51} \mathrm{H}_{44} \mathrm{~N}_{13} \mathrm{O}_{12} \mathrm{~S}_{6} 1222.1551, \Delta 1.13$ p.p.m.). Low resolution LC-MS data were measured using a Hewlett-Packard HP1100 integrated LC/MS system with a reversed-phase $\mathrm{C}_{18}$ column (Phenomenex Luna, $4.6 \mathrm{~mm} \times 100 \mathrm{~mm}, 5 \mathrm{~mm}$, Phenomenex, Torrance, CA, USA) at a flow rate of $0.7 \mathrm{ml} \mathrm{min}^{-1}$

\section{Bacterial strains and susceptibility testing}

Details on the strains used in this study are in Table 2. MRSA Sanger 252 USA 200 strain was obtained through the Network of Antimicrobial Resistance in S. aureus (NARSA) program supported under NIAID/NIH contract no. HHSN272200700055C. Susceptibility testing was performed in duplicate using cation-adjusted Mueller-Hinton broth (CA-MHB) and Mueller-Hinton agar
Table $1{ }^{1} \mathrm{H}$ and ${ }^{13} \mathrm{C}$ NMR spectral data of nosiheptide ( $\delta$ in p.p.m., $J$ in $\mathrm{Hz}$ )

\begin{tabular}{|c|c|c|c|c|}
\hline Ind $\mathrm{CO}$ & 180.69 & But 3 & 128.14 & $6.32(\mathrm{q}, J=6.84)$ \\
\hline Glu CO & 173.52 & Pyr 4 & 127.17 & $7.64(\mathrm{~s})$ \\
\hline $\operatorname{Thz}(3) 2$ & 170.18 & $\operatorname{Thz}(5) 5$ & 126.52 & $8.28(\mathrm{~s})$ \\
\hline Thz(4) 2 & 169.64 & Thz(1) 5 & 125.82 & $8.47(\mathrm{~s})$ \\
\hline Thr CO & 169.44 & $\operatorname{Thz}(3) 5$ & 125.67 & $8.18(\mathrm{~s})$ \\
\hline Thz(5) 2 & 166.83 & Ind 6 & 125.28 & $7.41(\mathrm{t}, J=7.57)$ \\
\hline $\operatorname{Thz}(2) 2$ & 166.41 & Ind 3a & 124.36 & \\
\hline Deala CO & 165.99 & Thz(2) 5 & 123.91 & $7.91(\mathrm{~s})$ \\
\hline $\operatorname{Thz}(1) 2$ & 164.85 & Ind 5 & 122.24 & $7.12(\mathrm{~d}, J=6.95)$ \\
\hline Thz(3) CO & 161.43 & Thz(4) 5 & 120.38 & $7.66(\mathrm{~s})$ \\
\hline $\mathrm{Thz}(2) \mathrm{CO}$ & 160.84 & Ind 3 & 118.90 & \\
\hline Thz(1) CO & 160.57 & Ind 7 & 115.31 & $7.79(\mathrm{~d}, J=7.04)$ \\
\hline Thz(5) CO & 158.59 & Deala 3 & 104.03 & $\begin{array}{l}6.60 E(\mathrm{~d}, J=1.89), \\
5.58 Z(\mathrm{~d}, J=1.89)\end{array}$ \\
\hline Thz(4) 4 & 155.00 & Thr 3 & 67.45 & $4.04(\mathrm{~m})$ \\
\hline Pyr 3 & 150.30 & Glu 4 & 67.18 & $4.14(\mathrm{~d}, J=11.54)$ \\
\hline $\operatorname{Thz}(1) 4$ & 149.81 & Ind 4' & 66.60 & $\begin{array}{l}5.85(\mathrm{~d}, J=11.25) \\
5.02(\mathrm{~d}, \mathrm{br}, J=10.79)\end{array}$ \\
\hline Thz(5) 4 & 149.76 & Thr 2 & 56.75 & $4.36(\mathrm{~d}, J=7.79)$ \\
\hline Thz(3) 4 & 148.45 & Cys 2 & 50.17 & $5.98(\mathrm{~d}, \mathrm{br}, J=9.00)$ \\
\hline $\operatorname{Thz}(2) 4$ & 147.86 & Glu 2 & 45.85 & $5.76(t, J=10.19)$ \\
\hline Pyr 6 & 143.99 & Glu 3 & 36.72 & $2.35 S(\mathrm{~m}), 1.80 R(\mathrm{~m})$ \\
\hline Ind $7 a$ & 137.44 & Cys 3 & 29.71 & $\begin{array}{l}3.79(\mathrm{dd}, J=3.15,12.06) \\
3.71(\mathrm{dd}, J=4.50,12.00)\end{array}$ \\
\hline Pyr 2 & 134.46 & $\mathrm{Thr}_{\mathrm{CH}}$ & 17.52 & $0.95(\mathrm{~s})$ \\
\hline Deala 2 & 132.94 & But $\mathrm{CH}_{3}$ & 14.58 & $1.68(\mathrm{~d}, J=7.00)$ \\
\hline Ind 2 & 130.64 & Ind $\mathrm{CH}_{3}$ & 11.96 & $2.54(\mathrm{~s})$ \\
\hline Pyr 5 & 130.44 & Ind $\mathrm{NH}$ & & $10.83(\mathrm{~s}, \mathrm{br})$ \\
\hline But 2 & 129.41 & Deala NH & & $9.99(\mathrm{~s})$ \\
\hline \multirow[t]{6}{*}{ Ind 4} & 128.73 & But NH & & $9.76(\mathrm{~s})$ \\
\hline & & Glu NH & & $7.97(\mathrm{~d}, \mathrm{br}, J=7.29)$ \\
\hline & & Glu OH & & not observed \\
\hline & & Cys NH & & $7.82(\mathrm{~s})$ \\
\hline & & Thr NH & & $7.50(\mathrm{~s})$ \\
\hline & & Thr OH & & $7.48(\mathrm{~s})$ \\
\hline
\end{tabular}

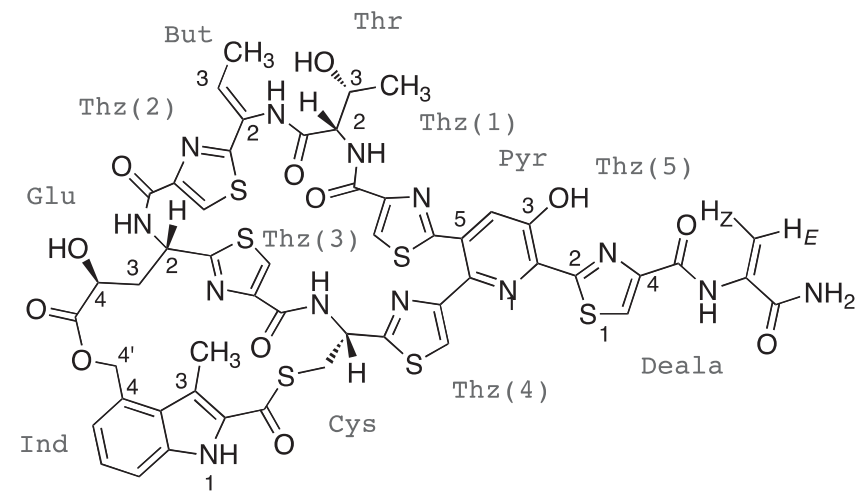

Figure 1 Structure of marine-derived nosiheptide. ${ }^{8}$ A full color version of this figure is available at The Journal of Antibiotics journal online.

according to Clinical and Laboratory Standards Institute methods. ${ }^{9}$ The MIC for Clostridium difficile was determined by the agar dilution reference method according to CLSI guidelines. ${ }^{10,11}$ Susceptibility of nosiheptide against MRSA strain TCH1516 was also determined in the presence of $20 \%$ activated-pooled human serum (freshly collected from consenting healthy donors) and 80\% MHB 
Table 2 Nosiheptide MIC

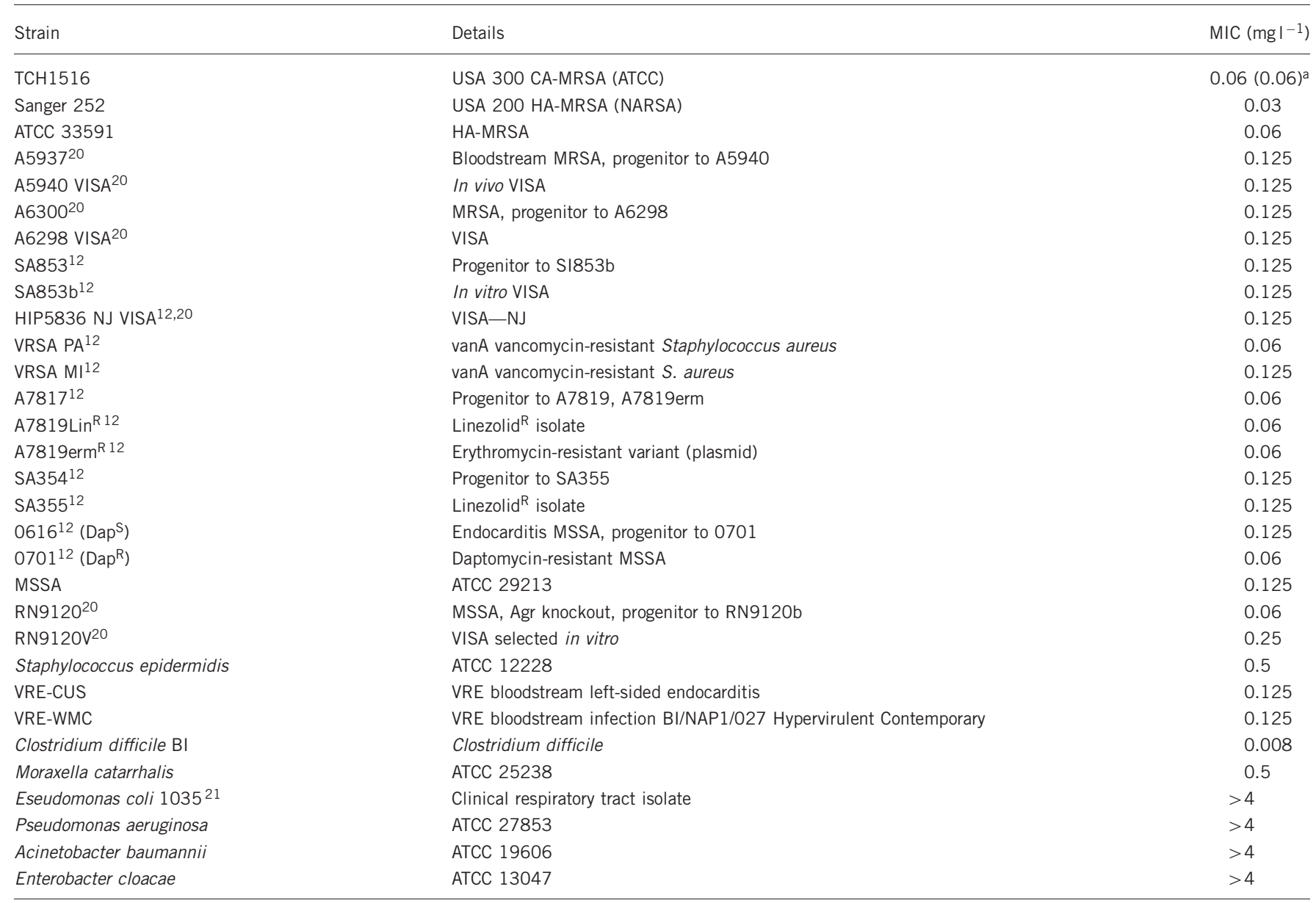

Abbreviations: ATCC, American Type Culture Collection; VRE-CUS, vancomycin-resistant Enterococcus faecium.

aMIC in $20 \%$ human serum.

by adding resazurin and assessing the conversion to resorufin exactly as previously described. ${ }^{12}$ This method provides a visual readout of color change from the blue indicator resazurin to the pink resorufin, a sign of bacterial growth.

\section{Time-kill studies}

Time-kill studies were done essentially as previously described in this laboratory. ${ }^{13}$ Briefly, nosiheptide was added at multiples of the MIC in CAMHB. MRSA strains or vancomycin-resistant Enterococcus faecium were added at a starting inoculum of $5 \times 10^{5} \mathrm{cfu} \mathrm{ml}^{-1}$, and the cultures were incubated in a $37^{\circ} \mathrm{C}$ shaking incubator (New Brunswick Scientific, Enfield, CT, USA). Samples were taken at specified timepoints for enumeration of viable bacteria by plating serial dilutions on Todd-Hewitt agar plates.

\section{Post-antibiotic effect}

PAE was assessed using the viable plate count method as previously described. ${ }^{13}$ For these studies, either nosiheptide or vancomycin was added at $10 \times$ their respective MICs to $14 \mathrm{ml}$ Falcon tubes containing CA-MHB. CAMRSA strain TCH1516 or HA-MRSA strain Sanger 252 was added at $5 \times 10^{5}$ cfu $\mathrm{ml}$, and the tubes were incubated under shaking conditions at $37^{\circ} \mathrm{C}$ for $1 \mathrm{~h}$. The bacteria were then pelleted and washed in $4 \mathrm{ml}$ of antibiotic-free CAMHB. The pellets were resuspended in $4 \mathrm{ml} \mathrm{CA-MHB}$ and incubated at $37^{\circ} \mathrm{C}$ in a shaking incubator. Surviving bacteria were assessed at specified timepoints as for the time-kill assay. The PAE was determined as previously described. ${ }^{13,14}$

\section{Mammalian cell cytotoxicity}

Mammalian cell cytotoxicity was assessed as previously described. ${ }^{13}$ Briefly, $2 \times 10^{4} \mathrm{HeLa}$ cells (American Type Culture Collection no. CCL-2, ATCC, Manassas, VA, USA) were seeded per well of sterile 96-well tissue culturetreated plates (Falcon; Becton Dickinson, Franklin Lakes, NJ, USA). After $24 \mathrm{~h}$, the medium (RPMI containing 10\% heat-inactivated fetal bovine serum) was replaced with fresh medium containing increasing concentrations of nosiheptide up to $128 \mathrm{mgl}^{-1}$, and the plates were incubated at $37^{\circ} \mathrm{C}$ in $5 \%$ $\mathrm{CO}_{2}$. Cell viability was assayed at $72 \mathrm{~h}$ by measuring the reduction of MTS (3-(4,5-dimethylthiazol-2-yl)-5-(3-carboxymethoxyphenyl)-2-(4-sulfophenyl)$2 \mathrm{H}$-tetrazolium) using the CellTiter 96 Aqueous nonradioactive cell proliferation assay according to the manufacturer's instructions (Promega, Madison, WI, USA).

\section{In vivo testing}

A murine model of i.p. infection was used to test in vivo efficacy of nosiheptide. ${ }^{15}$ Eight-week-old female CD1 mice (Charles River, Wilmington, MA, USA) were injected i.p. with $1-2 \times 10^{9} \mathrm{cfu}$ of the HA-MRSA strain Sanger 252 in $4 \%$ hog gastric mucin. The mice were treated with nosiheptide $\left(20 \mathrm{mg} \mathrm{kg}^{-1}\right)$ i.p. at 1 and $8 \mathrm{~h}$ after bacterial inoculation and were monitored for mortality and clinical status twice daily thereafter for 5 days. Moribund mice were humanely killed as were mice at the end of the study. These experiments were reviewed and approved by the Animal Subjects Committee of UCSD. 
Statistical analysis

The data from the survival studies were analyzed with GraphPad Prism (GraphPad Software, Incorporated, La Jolla, CA, USA) using log-rank analysis (Mantel-Cox test). Data were considered significant at $P<0.05$.

\section{RESULTS}

\section{In vitro antimicrobial activity}

Nosiheptide exhibited strong activity against all contemporary MRSA and MSSA strains tested including multidrug-resistant clinical isolates (Table 2), with MIC values $\leqslant 0.25 \mathrm{mgl}^{-1}$. Notably, clinical MRSA isolates previously demonstrated to have developed resistance to front-line antibiotics including daptomycin, linezolid and vancomycin remained highly sensitive to nosiheptide. This activity against MRSA was also completely unaffected by the presence of $20 \%$ human serum (Table 2). Nosiheptide was also highly active against Enterococcus spp. and the Gram-negative pathogen Moraxella catarrhalis, but did not possess activity at $\leqslant 4 \mathrm{mgl}^{-1}$ against the other Gram-negative strains tested including clinical isolates of Escherichia coli and Pseudomonas aeruginosa. Addressing another critical antibiotic resistance challenge, nosiheptide showed extremely potent activity against the contemporary hypervirulent BI strain of $C$. difficile (also known as the NAP1 or ribotype 027 strain).

\section{Time-kill kinetics}

In vitro time-kill analysis was used to assess nosiheptide killing kinetics. Nosiheptide was rapidly bactericidal against MRSA in a concentration- and time-dependent manner (Figure 2a), with a nearly 2-log kill noted within $6 \mathrm{~h}$ at both $10 \times$ and $20 \times$ MIC. A 2-log kill of MRSA was also noted for nosiheptide against MRSA resistant to other antibiotics, such as linezolid or erythromycin (Figure 2b). Although nosiheptide demonstrated bactericidal activity against MRSA at $10 \times$ and $20 \times$ the MIC, it was bacteriostatic against a clinical bloodstream isolate of vancomycin-resistant Enterococcus faecium up to $64 \times \mathrm{MIC}$ (Figure 2c). The MRSA killing kinetics of nosiheptide also compared favorably with vancomycin kinetics at $10 \times$ and $20 \times$ their respective MICs (Figure 2d).

\section{Prolonged PAE and lack of cytotoxicity}

Given its favorable MRSA killing kinetics, nosiheptide was assessed for its PAE. Notably, nosiheptide exhibited prolonged PAEs against both HA- and CA-MRSA compared with the most commonly used systemic antibiotic, vancomycin, at $10 \times$ their respective MICs (Figures $3 \mathrm{a}$ and $\mathrm{b}$ ). The PAE for nosiheptide was calculated to exceed $9 \mathrm{~h}$ for both HA- and CA-MRSA. We also tested nosiheptide for in vitro evidence of mammalian cell cytotoxicity as a prelude to our in vivo experiments. No evidence for nosiheptide cytotoxicity was observed when incubated for $72 \mathrm{~h}$ with the cervical carcinoma HeLa cell line at up to $128 \mathrm{mgl}^{-1}$, which is $\sim 1000$-fold above the MIC against MRSA. We also found that nosiheptide activity against USA300 MRSA was not inhibited in 20\% human serum (Table 2).

\section{In vivo activity}

Owing to its potent anti-MRSA activities in vitro, nosiheptide was tested in a murine model of i.p. MRSA infection. Mice were infected with HA-MRSA strain Sanger 252, followed by nosiheptide treatment $\left(20 \mathrm{mg} \mathrm{kg}^{-1}\right.$, i.p.) at 1 and $8 \mathrm{~h}$ post infection. Although all mice became lethargic and exhibited piloerection within $\sim 4 \mathrm{~h}$ of infection, nosiheptide provided significant $(P<0.03)$ protection against mortality (Figure 4 ). Ten out of 10 of the nosiheptide-treated mice remained alive on day 3 , whereas $6 / 10$ of the controls died on day 1 . By the end of the study, only one mouse in the nosiheptide group had died. These results provide evidence of significant in vivo activity for nosiheptide.

\section{DISCUSSION}

In our search for new anti-MRSA antibiotics from marine-derived microorganisms, we identified an actinomycete strain that produced a metabolite shown to be nosiheptide. Although the structure of this
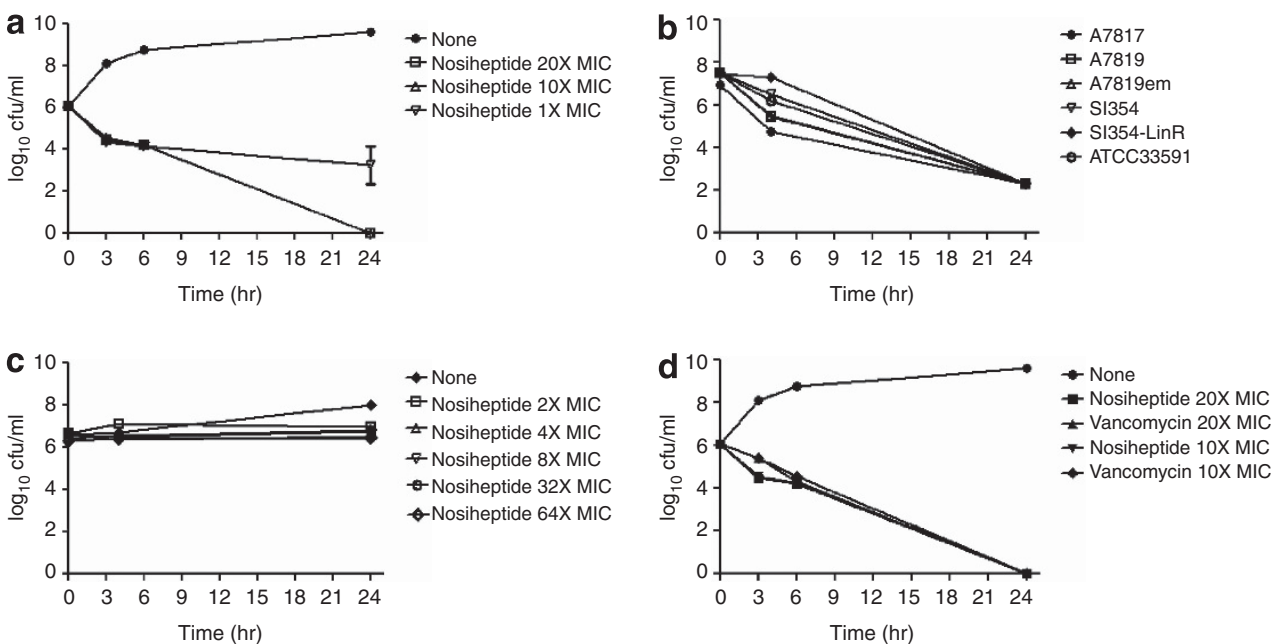

Figure 2 Time-kill kinetics of nosiheptide against MRSA and VRE. Nosiheptide killing kinetics was studied against MRSA and VRE at multiples of their MICs (MIC $=0.06 \mathrm{mgI}^{-1}$ for nosiheptide). (a) Killing kinetics of nosiheptide at $1 \times, 10 \times$ and $20 \mathrm{X}$ MIC against MRSA USA300 strain TCH1516 $\left(\mathrm{MIC}=0.06 \mathrm{mgl}^{-1}\right)$. (b) Nosiheptide killing kinetics against various multi-drug resistant MRSA at $10 \times$ their respective MICs. (c) Nosiheptide activity over time against vancomycin-resistant Enterococcus faecium at multiples of the MIC (MIC $=0.125 \mathrm{mg}^{-1}$ ). (d) Nosiheptide time-kill kinetics against MRSA USA300 strain TCH1516 compared with vancomycin at $10 \times$ and $20 \times$ their respective MICs $\left(\mathrm{MIC}=0.06 \mathrm{mgI}^{-1}\right.$ for nosiheptide; MIC $=1.56 \mathrm{mgI}^{-1}$ for vancomycin). Each assay was repeated in duplicate (for VRE) or triplicate (for MRSA), and the data shown are the mean \pm s.d. from one representative experiment. 
a

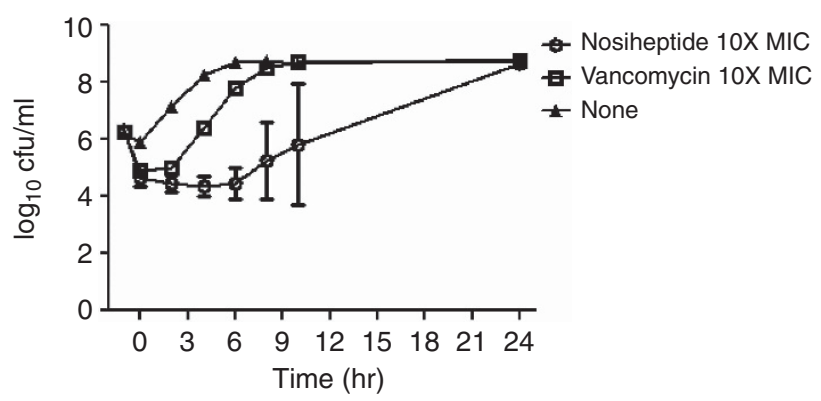

b

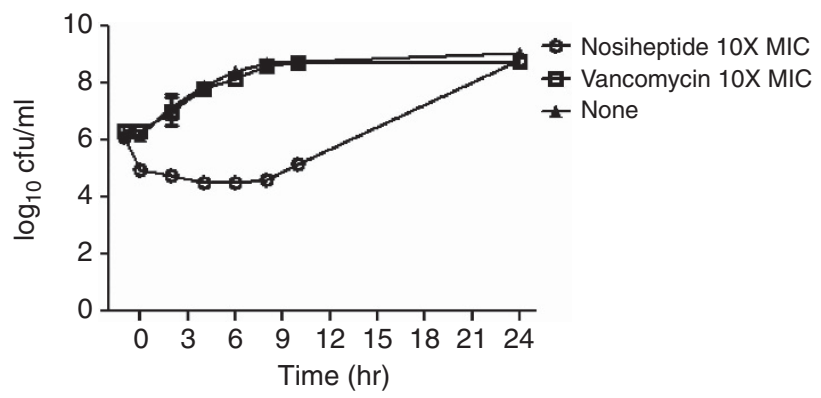

Figure 3 PAE of nosiheptide on contemporary MRSA strains. CA-MRSA strain TCH-1516 (a) or HA-MRSA strain Sanger 252 (b) were incubated for $1 \mathrm{~h}$ with nosiheptide or vancomycin at $10 \times$ MIC. The antibiotics were removed, and the bacteria were washed and allowed to recover in antibioticfree media. The curves show the rates of growth following antibiotic treatment. The data represent the mean \pm s.d. of one representative assay repeated in duplicate.

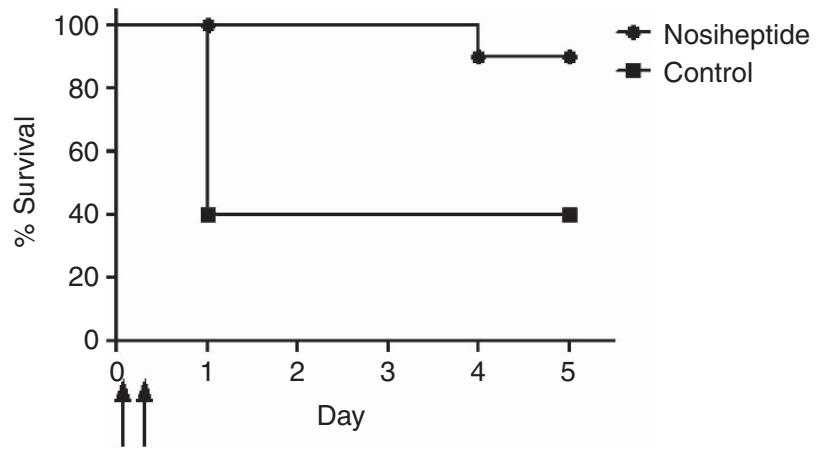

Figure 4 Nosiheptide protection in a murine model of i.p. MRSA infection. Plot showing survival of mice ( $n=10$ per group) infected i.p. with HAMRSA strain Sanger 252, followed by i.p. treatment with either nosiheptide (20 $\mathrm{mg} \mathrm{kg}^{-1}$, filled circles) or vehicle control (filled squares) at $1 \mathrm{~h}$ or $8 \mathrm{~h}$ after infection (represented by upward arrows on the $\mathrm{x}$ axis). $N=10$ mice per treatment group $(P<0.03$ by log-rank analysis).

compound was originally elucidated several decades ago and activity against Gram-positive activity was noted, a paucity of data exist on the therapeutic potential for this compound given contemporary concerns of multi-drug resistant bacterial pathogens. With growing antibiotic resistance exhibited by MRSA and other Gram-positive pathogens driving the need for new antimicrobials, we sought to further characterize this compound for its activity against HA- and CA-MRSA. Nosiheptide was exquisitely active against all contemporary MRSA strains tested regardless of their resistance to other front-line therapeutics including vancomycin, linezolid and daptomycin. Importantly, high-level activity was also noted against the increasingly problematic pathogen, $C$. difficile, especially significant as the FDA has approved only one new drug (Fidaxomicin) to treat $C$. difficile in the past two decades. Although the activity of some thiazole antibiotics may be reduced in the presence of serum, we found that nosiheptide activity against USA300 MRSA was unaffected in the presence of $20 \%$ human serum, suggesting that serum inhibition may not necessarily be a characteristic of all thiazole-containing antibiotics.

Nosiheptide exhibited favorable MRSA killing kinetics, a very prolonged PAE, and was active in a murine model of i.p. infection. Although in vivo evidence of nosiheptide activity has not been published, Benazet et al. ${ }^{16}$ report that nosiheptide protected mice from $S$. aureus mortality only when the compound was administered at the site of infection. However, no data were presented to support this claim. We report in the current paper nosiheptide activity when the compound is administered 1 and $8 \mathrm{~h}$ after MRSA injection at the site of infection as well (Figure 4). Our data are consistent with the claims of Benazet et al. ${ }^{16}$ and point to the fact that nosiheptide is efficacious in vivo but may either be metabolized or not distributed (due to localized precipitation or lack of absorption) when injected. A closely related compound, glycothiohexide $\alpha$, was once shown to be quite active against MRSA and vancomycin-resistant E. faecium. ${ }^{17-19}$ However, despite its in vitro potency, this compound had minimal activity in a $S$. aureus model of i.p. infection when administered s.c. It is unknown whether in the case of glycothiohexide $\alpha$, pharmacokinetic and/or possible metabolic factors abrogated potential efficacy. In pilot studies, we also found that nosiheptide exhibited significantly reduced activity when injected s.c. (data not shown) following i.p. MRSA inoculation and are currently embarking upon collaborative studies of liposomal and other lipophilic drug formulations to optimize nosiheptide delivery in vivo.

In sum, nosiheptide represents a promising antibiotic scaffold for further development owing to its potent anti-MRSA and other Grampositive activity, lack of inhibition by human serum, lack of mammalian cell cytotoxicity and demonstrated tolerability when delivered orally to animals. ${ }^{7}$ Many of the synthesis/manufacturing issues have already been addressed, as nosiheptide is produced in mass quantities by manufacturers worldwide. Cumulatively, these properties provide nosiheptide an advantage over other early-stage antibacterials, many of which are later found to be cytotoxic or serum-inhibited, or are fraught with difficulties in scale-up of production.

\section{ACKNOWLEDGEMENTS}

NMH was supported by the National Institutes of Health (NIH) Training Program in Marine Biotechnology (T32 GM067550) and a Ruth L Kirschstein National Research Service Award (NRSA) from National Institutes of Health grants (5 F31 GM090658-02). SL was supported by the German Research Foundation (DFG). WF, VN, PRJ and MEH were supported by National Institutes of Health grant GM084350. PRJ and WF acknowledge financial support from the NIH Fogerty Center International Cooperative Biodiversity Groups program (grant U01-TW00007-401). We thank K Freel and C Kauffman for assistance with fieldwork and W Aalbersberg (University of the South Pacific) for providing laboratory space and facilitating the field collections. We gratefully acknowledge the people of Fiji for their hospitality and permission to collect samples from their local waters. We are also grateful to Diane M Citron and Ellie Goldstein MD of RM Alden Research Laboratories (Culver City, CA, USA) for performing susceptibility testing against Clostridium difficile. 
1 Dhand, A. et al. Use of antistaphylococcal beta-lactams to increase daptomycin activity in eradicating persistent bacteremia due to methicillin-resistant Staphylococcus aureus: role of enhanced daptomycin binding. Clin. Infect. Dis. 53, 158-163 (2011).

2 Bagley, M. C., Dale, J. W., Merritt, E. A. \& Xiong, X. Thiopeptide antibiotics. Chem. Rev. 105, 685-714 (2005).

3 Nicolaou, K. C., Chen, J. S., Edmonds, D. J. \& Estrada, A. A. Recent advances in the chemistry and biology of naturally occurring antibiotics. Angew. Chem. Int. Ed. Engl. 48, 660-719 (2009)

4 Tanaka, T., Endo, T., Shimazu, A., Yoshida, R. \& Suzuki, Y. A new antibiotic, multhiomycin. J. Antibiot. 23, 231-237 (1970).

5 Tanaka, T., Sakaguchi, K. \& Yonehara, H. On the mode of action of multhiomycin, I. Effects of multhiomycin on macromolecular syntheses. J. Antibiot. 23, 401-407 (1970).

6 Cundliffe, E. \& Thompson, J. The mode of action of nosiheptide (multhiomycin) and the mechanism of resistance in the producing organism. J. Gen. Microbiol. 126, 185-192 (1981).

7 Benazet, F. \& Cartier, J. R. Effect of nosiheptide as a feed additive in chicks on the quantity, duration, prevalence of excretion, and resistance to antibacterial agents of Salmonella typhimurium; on the proportion of Escherichia coli and other coliforms resistant to antibacterial agents; and on their degree of resistance. Poult. Sci. 59, 1405-1415 (1980).

8 Mocek, $\mathrm{U}$. et al. ${ }^{1} \mathrm{H}$ and ${ }^{13} \mathrm{C}$ NMR Assignments of the thiopeptide antibiotic nosiheptide. J. Antibiot. 42, 1643-1648 (1989).

9 Clinical and Laboratory Standards Institute. Methods for Dilution Antimicrobial Susceptibility Tests for Bacteria that Grow Aerobically. Document M7-A7 (Clinical and Laboratory Standards Institute, Wayne, PA, 2006).

10 Clinical and Laboratory Standards Institute. Methods for Antimicrobial Susceptibility Testing of Anaerobic Bacteria. Approved Standard M11-A7 (Clinical and Laboratory Standards Institute, Wayne, PA, 2007).
11 Goldstein, E. J. C. et al. Comparative susceptibilities to fidaxomicin (OPT-80) of isolates collected at baseline, recurrence, and failure from patients in two Phase III trials of fidaxomicin against Clostridium difficile infection. Antimicrob. Agents Chemother. 55, 5194-5199 (2011)

12 Sakoulas, G. et al. Novel bacterial metabolite merochlorin A demonstrates in vitro activity against multi-drug resistant methicillin resistant Staphylococcus aureus. PloS One 7, e29439 (2012).

13 Haste, N. M. et al. Pharmacological properties of the marine natural product marinopyrrole A against methicillin resistant Staphylococcus aureus. Antimicrob. Agents Chemother. 55, 3305-3312 (2011).

14 Craig, W. A. \& Gundmundsson, S. in Antibiotics in Laboratory Medicine (ed. Lorian, V.) 4th edn. 296-329 (Williams and Wilkins, Baltimore, MD, 1996).

15 Haste, N. M. et al. Activity of the streptogramin antibiotic etamycin against methicillinresistant Staphylococcus aureus. J. Antibiot. 63, 219-224 (2010).

16 Benazet, F. et al. Nosiheptide, a sulfur-containing peptide antibiotic isolated from Streptomyces actuosus 40037. Experientia 36, 414-416 (1980).

17 Northcote, P. T. et al. Glycothiohexide alpha, a novel antibiotic produced by 'Sebekia' sp., LL-14E605. II. Isolation and physical-chemical characterization. J. Antibiot. 47, 894-900 (1994)

18 Northcote, P. T., Siegel, M., Borders, D.B. \& Lee, M.D. Glycothiohexide alpha, a nove antibiotic produced by 'Sebekia' sp., LL-14E605. III. Structural elucidation. J. Antibiot. 47, 901-908 (1994).

19 Steinberg, D. A. et al. Glycothiohexides, novel antibiotics produced by 'Sebekia' sp. LL14E605. I. Taxonomy, fermentation and biological evaluation. J. Antibiot. 47, 887-893 (1994)

20 Sakoulas, G. et al. Accessory gene regulator (agr) locus in geographically diverse Staphylococcus aureus with reduced susceptibility in vancomycin. Antimicrob. Agents Chemother. 46, 1492-1502 (2002).

21 Laplante, K. L. \& Sakoulas, G. Evaluating aztreonam and ceftazidime pharmacodynamics with Escherichia coli in combination with daptomycin, linezolid, or vancomycin in an in vitro pharmacodynamic model. Antimicrob. Agents Chemother. 53, 4549-4555 (2009). 